\title{
Vitamin D Supplementation Efficacy and Its Relationship to Grip Strength and Gait Speed in the Oldest Olds with Functional Dependence
}

\author{
Małgorzata Kupisz-Urbańska*1, Elżbieta Kozak-Szkopek², Krzysztof Galus² and Ewa Marci- \\ nowska-Suchowierska ${ }^{1}$
}

${ }^{1}$ Centre of Postgraduate Medical Education in Warsaw, Poland

${ }^{2}$ Medical University of Warsaw, Poland

*Corresponding author: Małgorzata Kupisz-Urbańska, Department of Geriatrics, Centre of Postgraduate Medical Education in Warsaw, Poland

ARTICLE INFO
Received: 幽 March 10, 2021
Published: March 22, 2021

Published: March 22, 2021

Citation: M Kupisz-Urbańska, E Kozak-Szkopek, K Galus, E Marcinowska-Suchowierska. Vitamin D Supplementation Efficacy and Its Relationship to Grip Strength and Gait Speed in the Oldest Olds with Functional Dependence. Biomed J Sci \& Tech Res 34(4)-2021. BJSTR. MS.ID.005583.

Keywords: Vitamin D Supplementation; Oldest Olds; Physical Performance; Handgrip Strength

Abbreviations: TUGT: Timed Up and Go Test; PTH: Parathyroid Hormone; EWGSOP2: European Working Group on Sarcopenia in Older People

\author{
ABSTRACT
}

Vitamin D deficiency is common in older people and oral vitamin D supplementation is considered safe. The main aim of this study was to evaluate the efficacy of vitamin D supplementation and its relationship to muscle strength and function in the oldest of the olds with functional decline. A total of 44 nursing home residents (mean age 85.1 years) participated in the study. The serum concentrations of 25-hydroxycholecalciferol $(25(\mathrm{OH}) \mathrm{D})$ and parathyroid hormone were measured at the beginning of the study, at three months and at six months. Based on their initial 25(OH)D measurement, the subjects were divided into two subgroups: one with 21 patients with $25(\mathrm{OH}) \mathrm{D}<10 \mathrm{ng} / \mathrm{ml}$ and the other with 23 patients with $25(\mathrm{OH}) \mathrm{D} \geq 10 \mathrm{ng} / \mathrm{ml}$. All the participants followed a three-month oral calciferol supplementation schedule. At the beginning of the study and after three months of vitamin D supplementation, all the participants had their handgrip strength assessed and were administered the timed up and go test (TUGT). For the whole group, the mean (SD) 25(OH)D serum concentration at the beginning of the study was 10.10 (6.07) ng/ml (8.11 (1.01) ng/ml in the first subgroup and 15.92 (9.02) ng/ml in the other); after three months of calciferol supplementation it was $32.05(6.61) \mathrm{ng} / \mathrm{ml}$ $(\mathrm{p}<0.001)$. No relationship was found between vitamin D status and handgrip strength nor TUGT. Vitamin D supplementation is effective to increase in 25(OH)D serum levels in elderly patients with functional decline. However, no relationship between vitamin D concentration and muscle strength and function was observed.

\section{Introduction}

Vitamin D is a well-known regulatory factor, not only in calcium and phosphorus homeostasis, but also in extraskeletal actions. It contributes to tissue health, reduces telomere-shortening rate, reduces cancer risk and appears to influence the optimal ageing process $[1,2]$. Moreover, vitamin D deficiency is common amongst elderly people, is linked to many chronic illnesses and its receptor expression in muscles decreases with age $[3,4]$. 7-Dehydrocholesterol concentration, the substrate for UV-induced skin vitamin D synthesis, is significantly reduced in the elderly when compared to young adults [5]. In addition, nutrition intake, calcium absorption, renal 1-alpha-hydroxylase activity and vitamin $\mathrm{D}$ receptors in different tissues decline with advanced age $[6,7]$. Evidence from different countries has highlighted vitamin D deficiency as a prevalent public health problem, but data on the vitamin status in older adults are insufficient $[8,9]$. In clinical practice, measurement of the total serum 25-hydroxycholecalciferol (25(OH)D) level is recommended to determine vitamin D status. Oral vitamin D supplementation is considered safe, well founded and recommended for use all over the world [10]. 
The population of geriatric patients is usually characterised by the presence of geriatric giants. In clinical practice, the evaluation of new geriatric giants such as sarcopenia could prove to be a crucial augmentation since it represents a powerful predictor of functional decline and poor patient outcome. In 2019, Cruz-Jentoft et al. published a revised European consensus on the definition and diagnosis of sarcopenia. The authors of these guidelines emphasised that focusing on low muscle strength - to detect cases of sarcopenia - and on poor physical performance - to identify sarcopenia severity - could be useful not only for scientific researchers but also for healthcare professionals. The European Working Group on Sarcopenia in Older People (EWGSOP2) also provided clear cut-off points for measurements, such as the timed up and go test (TUGT) and handgrip strength, that identify and characterise sarcopenia [11]. The main aim of this study was to evaluate the efficacy of vitamin D supplementation and its relationship to muscle strength and function in the oldest of the olds with functional decline.

\section{Materials and Methods}

The study group consisted of 32 women and 12 men aged between 75 and 98 years with a mean age of 85.1 years. All the subjects were nursing home residents with similar levels of sun exposure and diets that contained about $700 \mathrm{mg}$ of calcium. They were characterised by functional dependence with scores in the Activities of Daily Living test (Barthel scale) below 40 points. All participants were suffering from vitamin D deficiency or insufficiency coexisting with comorbidity: 18 subjects were diagnosed with heart failure, 19 subjects with hypertension, 29 subjects with other cardiovascular diseases, six subjects with pulmonary diseases, 13 subjects with diabetes, 16 subjects with gastritis, 27 subjects with osteoarthritis and 25 subjects with lower renal filtration. The exclusion criteria applied were advanced heart failure (NYHA class IV) and severe liver or kidney failure. The Bioethics Committee of the Medical University of Warsaw approved the study. This study was conducted over a 6-month period from July 2016 to January 2017. The protocol involved taking blood samples three times during the study: at the beginning, at three months and at six months. The serum concentrations of 25(OH)D, calcium and Parathyroid Hormone (PTH) were measured in all subjects at the beginning of the study. The initial serum vitamin D level was used as the indicator to divide the patients into two subgroups based on their 25(OH)D blood concentration. The first subgroup consisted of 21 patients with a 25(OH)D serum concentration below $10 \mathrm{ng} / \mathrm{ml}$. The other subgroup consisted of 23 patients with a 25(OH)D serum concentration equal to or above $10 \mathrm{ng} / \mathrm{ml}$. All the participants followed a three-month oral calciferol supplementation schedule.

The participants in the subgroup with 25(OH)D blood concentration lower than $10 \mathrm{ng} / \mathrm{ml}$ received $4000 \mathrm{IU}$ of calciferol daily for one month and 2000 IU daily for the next two months. The participants in the subgroup with 25(OH)D blood concentration higher than or equal to $10 \mathrm{ng} / \mathrm{ml}$ received $2000 \mathrm{IU}$ of calciferol daily for three months. The 25(OH)D serum level was then measured after three months of vitamin D supplementation. The third and final measurement of the serum level of 25(OH)D took place after another three months (during which vitamin D supplementation was no longer administered), i.e. six months after the start of the study. PTH was measured at the beginning of the study and after three months of vitamin D supplementation. At the beginning of the study, only one participant took over-the-counter vitamin D supplementation; all the other participants were prescribed vitamin D supplementation. At the beginning of the study, according to the recommendations of the Polish Society of Physiotherapy, all the participants had their handgrip strength assessed and were administered the TUGT. Following three months of vitamin D supplementation, the same tests were conducted. Characteristics of the study group are detailed in Table 1 . The 25(OH)D serum concentration was measured by chemiluminescent immunoassay and a Liaison analyser. The data were analysed by the Pearson correlation coefficient using R 3.4.1 (R Core Team (2017). R: A language and environment for statistical computing. $\mathrm{R}$ Foundation for Statistical Computing, Vienna, Austria). $\mathrm{p}<0.05$ was considered to be statistically significant.

Table 1: Characteristics of the study group.

\begin{tabular}{|c|c|}
\hline \multicolumn{2}{|c|}{ Characteristics } \\
\hline Number of subjects, $N$ & 44 \\
\hline Sex & \\
\hline Women (\%) & $32(72.7)$ \\
\hline Men (\%) & $12(27.3)$ \\
\hline Nursing home stay, years, mean [min/max] & $1.50[0.50,3.00]$ \\
\hline Age, years, mean (SD) & $85.10(7.20)$ \\
\hline Body mass, kg, mean (SD) & $64.44(13.07)$ \\
\hline Height, cm, mean (SD) & $151.43(11.34)$ \\
\hline Body mass index, BMI, mean (SD) & $28.40(5.54)$ \\
\hline Arm circumference, cm, mean (SD) & $26.39(3.82)$ \\
\hline Calf circumference, cm, mean (SD) & $32.47(3.55)$ \\
\hline
\end{tabular}

\section{Results}

For the whole group, the first measurement of vitamin D status (taken at the beginning of the study) determined from the $25(\mathrm{OH})$ D serum concentration was 10.10 (6.07) ng/ml (mean (SD)). Specifically, it was $8.11(1.01) \mathrm{ng} / \mathrm{ml}$ in the subgroup with initial vitamin 25(OH)D status below $10 \mathrm{ng} / \mathrm{ml}$ and 15.92 (9.02) ng/ml in the subgroup with initial vitamin $25(\mathrm{OH}) \mathrm{D}$ status equal to or above $10 \mathrm{ng} / \mathrm{ml}$. For the second measurement (taken after three months of vitamin D supplementation), the mean (SD) 25(OH) D serum level for all the subjects was significantly higher -32.05 (6.61) $\mathrm{ng} / \mathrm{ml}, \mathrm{p}<0.001$ - with the median being above $31 \mathrm{ng} / \mathrm{ml}$ for each subgroup. For the third measurement (taken after another three months during which vitamin D supplementation was no 
longer administered), the mean (SD) 25(OH)D serum level was significantly lower - 21.65 (6.28) ng/ml, p<0.001 (this trend was observed in both subgroups). The data are presented in Figure 1. A statistically significant correlation was found in the vitamin D status difference between the first and the second measurement; the correlation factor being $-0.63, \mathrm{p}<0.001$. The mean (SD) PTH serum concentration at the beginning of the study was 61.35 (26.34) $\mathrm{mg} /$ $\mathrm{ml}$. After three months of vitamin D supplementation, it increased to 68.16 (34.14) $\mathrm{mg} / \mathrm{ml}$, without statistical significance $(\mathrm{p}=0.06)$. A statistically significant difference was found between the two subgroups at the beginning of the study: the mean (SD) PTH serum concentration was $88.10(51.83) \mathrm{mg} / \mathrm{ml}$ in the first subgroup and
$55.10(18.23) \mathrm{mg} / \mathrm{ml}$ in the other subgroup, $\mathrm{p}<0.01$. This was also the case after three months of vitamin D supplementation: 86.69 (43.45) $\mathrm{mg} / \mathrm{ml}$ in the first subgroup and 57.13 (22.10) $\mathrm{mg} / \mathrm{ml}$ in the other subgroup, $\mathrm{p}<0.01$. No statistically significant correlation was observed between vitamin $\mathrm{D}$ concentrations and PTH levels. To investigate the relationship between vitamin D status and physical performance, the TUGT and handgrip strength were compared before and after vitamin D supplementation. No significant difference was observed in the patients' walking speed or muscle strength. There was no correlation between the $25(\mathrm{OH})$ D concentration and handgrip strength nor between the 25(OH)D concentration and the TUGT. The data are presented in Tables $2 \& 3$.
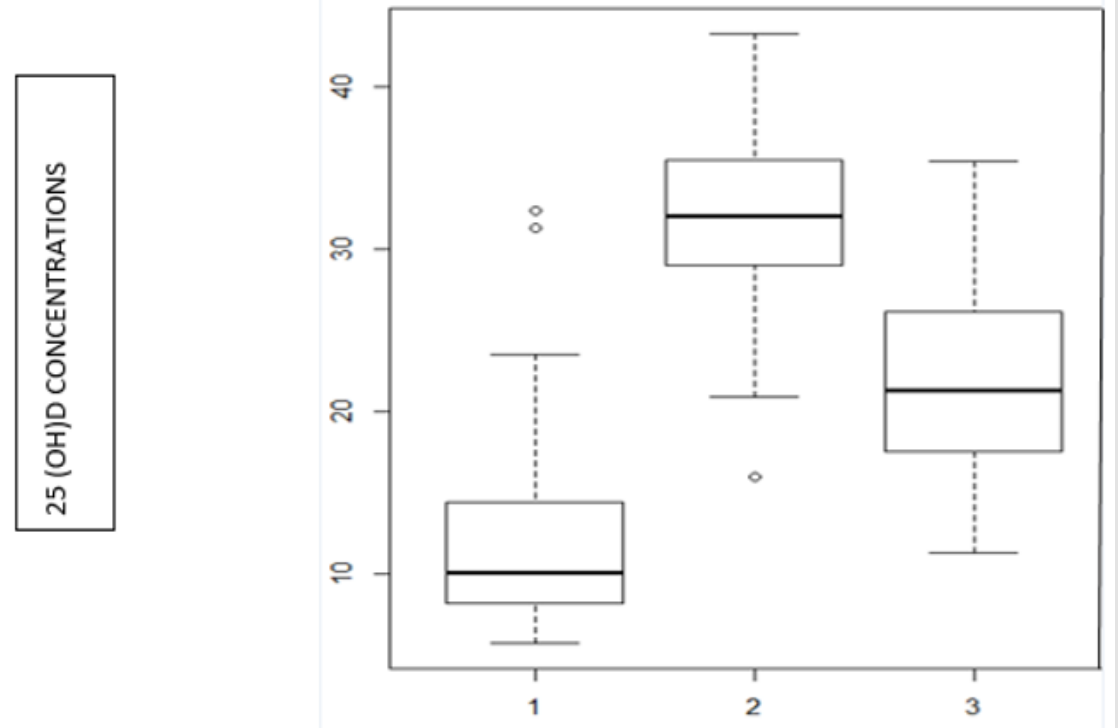

Figure 1: 25(OH)D serum concentrations $(\mathrm{ng} / \mathrm{ml})$ of nursing home residents before and after vitamin D supplementation (at the beginning of the study [1], after three months of vitamin D supplementation [2], after another three months during which vitamin D supplementation was no longer administered [3]).

Table 2: Comparison of handgrip strength and timed up and go test (TUGT) at the beginning of the study and after three months of vitamin D supplementation ( $\mathrm{t}$ test).

\begin{tabular}{|c|c|c|c|}
\hline & $\begin{array}{c}\text { Measurements at the Beginning of } \\
\text { the Study }\end{array}$ & $\begin{array}{c}\text { Measurements after Three Months of } \\
\text { Vitamin D Supplementation }\end{array}$ & p Value \\
\hline $\begin{array}{c}\text { HANDGRIP STRENGTH FOR RIGHT } \\
\text { HAND, kg, mean (SD) } \\
\text { Min-Max }\end{array}$ & $14.5(4.8)$ & $14.8(5.3)$ & $2-26$ \\
\hline $\begin{array}{c}\text { HANDGRIP STRENGTH FOR LEFT } \\
\text { HAND, kg, mean (SD) }\end{array}$ & $5-27$ & $12.6(5.5)$ & $\mathrm{p}$ \\
\hline Min-Max & $12.9(6.8)$ & $4-26$ & $\mathrm{p}=0.464$ \\
\hline TUGT, s, mean (SD) & $1-24$ & $30.9(15.4)$ & $\mathrm{p}=0.724$ \\
\hline
\end{tabular}


Table 3: Correlation of $25(\mathrm{OH}) \mathrm{D}$ serum concentration with handgrip strength and $25(\mathrm{OH}) \mathrm{D}$ serum concentration with the timed up and go test (TUGT) at the beginning of the study and after three months of vitamin D supplementation.

\begin{tabular}{|c|c|c|c|c|}
\hline \multirow{2}{*}{ Variable } & \multicolumn{2}{|c|}{$\begin{array}{c}\text { Correlation Factor with 25(OH)D at the Beginning of } \\
\text { the Study }\end{array}$} & $\begin{array}{c}\text { Correlation Factor with 25(OH)D after Three Months } \\
\text { of Vitamin D Supplementation }\end{array}$ \\
\cline { 2 - 5 } & Correlation Factor & p value & Correlation Factor & p Value \\
\hline $\begin{array}{c}\text { Handgrip Strength for Right } \\
\text { Hand }\end{array}$ & 0.332 & 0.078 & 0.21 & 0.24 \\
\hline $\begin{array}{c}\text { Handgrip Strength for Left } \\
\text { Hand }\end{array}$ & 0.311 & 0.089 & 0.329 & 0.061 \\
\hline TUGT* & 0.267 & 0.116 & 0.094 & 0.602 \\
\hline
\end{tabular}

Note: *TUGT- timed up and go test

\section{Discussion}

Our data suggest that, in the oldest of elderly nursing home residents, a three-month vitamin $\mathrm{D}$ supplementation is sufficient to achieve a normal serum concentration of 25(OH)D. A higher increase in vitamin D status was observed in the subgroup with an initial 25(OH)D serum concentration below $10 \mathrm{ng} / \mathrm{ml}$. This finding is in accordance with literature data [12-15]. In Poland, contrary to other countries, not only is the serum concentration of 25(OH)D very low in nursing home residents, but the level of supplementation also appears to be insufficient; only one of the participants in our study was being supplemented with a dose of 2000 IU vitamin D per day. Furthermore, the process of fortifying food with vitamin D is not practiced in Poland. At the same time, low levels of outdoor activity combined with reduced skin production in the elderly likely constitute crucial factors of vitamin D deficiency. Our study also revealed that, in elderly subjects with functional decline (a common characteristic for nursing home residents), 25(OH)D levels are lower than in younger groups without physical decline. Płudowski et al. reported that the lowest mean of vitamin D status in the Polish urban adult population is about $15 \mathrm{ng} / \mathrm{ml}$. Additionally, in populations over 80 years of age, the mean vitamin D status is 17.5 $\mathrm{ng} / \mathrm{ml}$, higher than in our study [16]. Taking into account the lower 25(OH)D concentration after three successive months without supplementation, sufficient vitamin D supplementation should be systematically applied to achieve permanent optimal blood $25(\mathrm{OH})$ D concentrations necessary for skeletal and extraskeletal actions. Nonetheless, at the time this study was conducted, the Polish guidelines for vitamin D supplementation were not well established and as a consequence may be the main reason for the low compliance of the recommendations for vitamin D supplementation [17].

Moreover, optimal vitamin D blood concentration can be defined by several criteria, for example PTH. In a global study, certain correlation trends with PTH have been found in subgroups with an initial lower vitamin D status and significantly higher PTH [18]. It is important to take into account that for PTH stabilisation a period of about 12 months is necessary. In the present study, significant correlations were not found between vitamin D status and PTH. It is likely that the small number of participants, their comorbidity, as well as the high standard deviation of the PTH values substantially contributed to the background of multifactorial mechanisms influencing the relationship between vitamin D metabolites and PTH $[19,20]$. In contrast, a study by Fisher et al. reported that the only predictor of hip fracture was elevated serum PTH inversely correlated with vitamin D status [21]. In our study, evaluation of the relationship between vitamin D status and physical performance revealed that three months of supplementation had no impact on muscle strength and muscle function measured by the TUGT. Furthermore, no correlations were found between vitamin D status and handgrip strength or muscle function in both subgroups at the beginning of the study nor after three months of supplementation. It should be mentioned that all nursing home residents in Poland are encouraged to partake in long-term physiotherapy. It is possible that the very poor initial physical performance of our subjects (only one person completed the TUGT within the normal time) contributed to the lack of pleiotropic effects of vitamin D supplementation. In line with our data, Matheï et al. reported no relation between vitamin D status and physical performance in octogenarians [22]. Conversely, studies by Jing et al. and Aspell et al. in younger subjects reported that vitamin D status was related to handgrip strength and physical performance $[23,24]$.

This finding raises the possibility that in advanced age, despite the observed changes in laboratory tests, the impact on clinical symptoms differs from younger groups. Additionally, as opposed to younger and healthier populations, the duration of vitamin D deficiency in the oldest olds may be prolonged enough to diminish potential correlations [25]. Thus, it should be taken into account that probably not all elderly people will benefit from vitamin D supplementation when considering poor physical function and functional decline. Currently, the evaluation, prevention and treatment of vitamin D deficiency appear to be well established; however, how this influences the clinical outcome of the oldest olds remains an issue of debate [26-28]. The primary limitation of this study was the small number of participants, making it impossible to create a clearly homogeneous population. The second limitation was the study's short duration which prohibited the evaluation of longterm supplementation effects. In addition, it should be highlighted that our study was carried out before the Polish guidelines for 
vitamin D supplementation were published. Therefore, not every nursing home was conducting and maintaining sufficient vitamin D supplementation. In Poland, as in all other countries, the number of the very oldest nursing home residents will increase in the coming years. This particular population is of special interest as it may be representative of patients with unsuccessful ageing processes.

\section{Conclusion}

Our study presents data indicating that vitamin D supplementation is effective amongst advanced age patients with comorbidity and functional decline. However, no relationship between vitamin D status and sarcopenia characteristics (muscle strength and function) was observed. Further studies are needed to fully assess the clinical impact of vitamin D supplementation in subjects ageing unsuccessfully.

\section{Contribution Statement}

MKU contributed to the design and implementation of the research, to the analysis of the results, and to the writing of the manuscript. EKS and KG contributed to the design and implementation of the research. EMS contributed to the analysis of the results and to the final revision of the manuscript.

\section{Declaration of Conflict of Interest}

None.

\section{References}

1. Siebert C, Dos Santos TM, Bertó CG, Parisi MM, Coelho RP, et al. (2018) Vitamin D Supplementation Reverses DNA Damage and Telomeres Shortening Caused by Ovariectomy in Hippocampus of Wistar Rats. Neurotox Res 34: 538-546.

2. Berridge MJ (2017) Vitamin D deficiency accelerates ageing and agerelated diseases: a novel hypothesis. J Physiol 595: 6825-6836.

3. Dudenkov DV, Mara KC, Petterson TM, Maxson JA, Thacher TD (2018) Serum 25-Hydroxyvitamin D Values and Risk of All-Cause and CauseSpecific Mortality: A Population-Based Cohort Study. Mayo Clin Proc 93(6): 721-730.

4. Bischoff-Ferrari HA, Borchers M, Gudat F, Durmuller U, Stahelin HB, et al (2004) Vitamin D receptor expression in human muscle tissue decreases with age. J Bone Miner Res 19(2): 265-226.

5. MacLaughlin J, Holick MF (1985) Aging decreases the capacity of human skin to produce vitamin D3. J Clin Invest 76(4): 1536-1538.

6. Galagher JCH (2013) Vitamin D and aging. Endocrinol Metab Clin North Am 42(2): 319-332.

7. Veldurthy V, Wei R, Oz L, Dhawan P, Jeon YH, et al. (2016) Vitamin D, calcium homeostasis and aging. Bone Res 4: 16041.

8. Yanhul L, Xiaomin F, Zhang L, Liu M, Cheng X, et al. (2011) Effects of stratified vitamin D supplementation in middle age and elderly individuals with vitamin D insufficiency. End Care 4: 747-754.

9. Casey C, Woodside JV, Mc Ginty A, Young IS, Mcpeake J, et al. (2018) Factors associated with serum 25-hydroxyvitamin D concentrations in older people in Europe: the EUREYE study. Eur J Clin Nutr 73: 319-328.

10. Bouillon R (2017) Comparative analysis of nutritional guidelines for vitamin D. Nat Rev Endocrinol 13: 466-479.

11. Cruz-Jentoft AJ, Bahat G, Bauer J, Boirie Y, Bruyere O, et al. (2019) Sarcopenia: revised European consensus on definition and diagnosis. Age Ageing 48(1): 16-31.
12. Vaes AMM, Tieland M, De Regt MF, Wittwer J, Van Loon LJC, et al. (2017) Dose-response effects of supplementation with calcifediol on serum 25-hydroxyvitamin D status and its metabolites: A randomized controlled trial in older adults. Clin Nutr 37(3): 808-814.

13. Pietras SM, Obayan BK, Cai MH, Holick MF (2009) Vitamin D2 treatment for vitamin D deficiency and insufficiency for up to 6 years. Arch Inter Med 169(19): 1806-1808.

14. Holick MF, Chen TC (2008) Vitamin D deficiency: A worldwide problem with health consequences. Am J Clin Nutr 87: 1080S-1086S.

15. Brenner H, Jansen KU, Saum KU (2017) Supplementation trials aimed at reducing mortality have much higher power when focussing on people with low serum 24-hydroxyvitamin D levels. B J Nutr 147: 1325-1333.

16. Płudowski P, Ducki C, Konstantynowicz J, Jaworski M (2016) Vitamin D status in Poland. Pol Arch Med Int 126: 530-539.

17. Rusińska A, Płudowski P, Walczak M, Borszewska-Kornacka MK, Bossowski A, et al. (2018) Vitamin D Supplementation Guidelines for General Population and Groups at Risk of Vitamin D Deficiency in PolandRecommendations of the Polish Society of Pediatric Endocrinology and Diabetes and the Expert Panel With Participation of National Specialist Consultants and Representatives of Scientific Societies-2018 Update. Front Endocrinol 9: 246.

18. Lips P, Duong T, Oleksik A, Black D, Cummings S, et al. (2001) A global study of vitamin D status and parathyroid function in po stmenopausal women with osteoporosis: baseline data from the multiple outcomes of raloxifene evaluation clinical trial. J Clin Endocrinol Metab 86(3): 12121221.

19. Chang WT, Wu CH, Hsu LW, Chen PW, Yu JR, et al. (2017) Serum vitamin D, intact parathyroid hormone, and Fetuin A concentrations were associated with geriatric sarcopenia and cardiac hypertrophy. Sci Rep 7(1): 40996.

20. Nuti R, Merlotti D, Gennari L (2011) Vitamin D deficiency and primary hyperparathyroidism. J Endocrinol Invest 34(7): 45-49.

21. Fisher A, Goh S, Srikusalanukul W, Davis M (2009) Elevated Serum PTH Is Independently Associated with Poor Outcomes in Older Patients with Hip Fracture and Vitamin D Inadequacy. Calcified Tissue International 85: 301-309.

22. Matheï C, Van Pottelbergh G, Vaes B, Adriaensen W, Gruson D, et al. (2013) No relation between vitamin D status and physical performance in the oldest old: results from the Belfrail study. Age Ageing 42(2): 186190.

23. Jing W, Xuena W, Yeqing G, Liu M, Chi VTQ et al. (2019) Vitamin D Is Related to Handgrip Strength in Adult Men Aged 50 Years and Over: A Population Study From the TCLSIH Cohort Study. Clin Endocrinol (Oxf) 90(5): 753-765.

24. Aspell N, Laird E, Healy M, Lawlor B, O’Sullivan M (2019) Vitamin D Deficiency Is Associated With Impaired Muscle Strength And Physical Performance In Community-Dwelling Older Adults: Findings From The English Longitudinal Study Of Ageing. Clin Interv Aging 14: 1751-1761.

25. Majed B, MZ, Haider SS, Mustafa G (2016) Effects of Vitamin D supplementation on physical activity of patients with Heart Failure. Pak J Med Sci 32(6): 1430-1439.

26. Holick MF, Binkley NC, Bischoff-Ferrari HA, Gordon CM, Hanley DA, et al. (2011) Evaluation, treatment and prevention of vitamin D deficiency: An endocrine society clinical practice guideline. J Clin Endocrionol Metab 96(7): 1911-1930.

27. Reinmark L (2011) Effects of Vitamin D on muscle function and performance: A review of evidence from randomised controlled trials. Ther Adv Chronic Dis 2(1): 25-37.

28. Gkekas NK, Anagnostis P, Paraschou V, Stamiris D, Dellis S, et al. (2021) The effect of vitamin D plus protein supplementation on sarcopenia: A systematic review and meta-analysis of randomized controlled trials. Maturitas 145: 56-63. 
ISSN: 2574-1241

DOI: 10.26717/BJSTR.2021.34.005583

Małgorzata Kupisz-Urbańska. Biomed J Sci \& Tech Res

(c) This work is licensed under Creative

Submission Link: https://biomedres.us/submit-manuscript.php

\begin{tabular}{ll} 
BIOMEDICAL & Assets of Publishing with us \\
RESEARCHES & - Global archiving of articles \\
- Immediate, unrestricted online access & - Rigorous Peer Review Process \\
\hline
\end{tabular}

